\title{
Load Balancing in WSN using Node Degree Ratio based Algorithm
}

\author{
Vatsla Chauhan \\ Department of Computer Science \\ Ganpati Institute of Technology \& Management \\ Bilaspur, Haryana, India
}

\author{
Narender Singh \\ Department of Computer Science \\ Ganpati Institute of Technology \& Management \\ Bilaspur, Haryana, India
}

\begin{abstract}
A sensor network is a network with large number of sensors nodes. Each sensor node is defined with some energy parameters. The energy of every node is limited and irreplaceable. With each communication over the network some amount of energy is lost. One of network architecture defined in sensor network is the multiple gateway based architecture. According to this architecture, complete network is divided in smaller area segments and each segment is controlled by a gateway node. Sensor nodes communicate with the base station via the gateway. Each node can be defined in sensing range of one or more gateway nodes. One of the challenging tasks in such network is the effective deployment of nodes over the network. These sensor nodes are defined with energy specification. While defining these network types, the protocol specification is required. This protocol is actually responsible for the communication over the senor network. In this paper, We propose an algorithm for an improved load balanced gateway architecture. A restriction is imposed on a particular type of nodes present in the cluster. An energy effective load balancing approach is suggested to generate the gateway network and to control the communication over the network. The selection of gateway is done using the energy and probability parameters.
\end{abstract}

\section{Keywords}

Wireless sensor network, Node degree, Gateway, Ratio analysis, Connectivity ratio

\section{INTRODUCTION}

Sensor network is defined as an organized adhoc network in which large number of sensor nodes and the resources are combined to perform the communication. These networks are generally defined with limited resources such as battery, bandwidth, and power supply [8]. It is considered as the most sensitive research area with the specification of physical environment as a wide range of communication applications comes under sensor network. The constraints of sensor network include the memory, computing power, energy etc. To perform the communication over the network, one of common adaptive approach is aggregation. According to this approach, the data is transferred from one node to other in a series till the destination node is not arrived. There are a number of aggregation approaches defined under network capabilities to perform the communication over the network nodes. These nodes are defined under the physical and environmental conditions such as humidity, temperature, pressure, vibration etc. In WSNs, each sensor node has various sensors depending on the WSN application. Each sensor nodes is able to perform the communication under energy constraint. It means each participating nodes gives some energy loss. This loss depends on the role of the node in communication. A communicating node gives different level of energy loss based on the operation such as transmission of data, receiving, forwarding or aggregating data. The communication in such network can be single hop or multihop. The data transmitted from these nodes is collected by the base station. The connecting network can also include laptop, PC or PDA. Senor network is defined with 3 main components:

- Node itself that sense the environment or the resource

- Another component is process component to perform the communication

- Communication components are responsible for the information exchange.

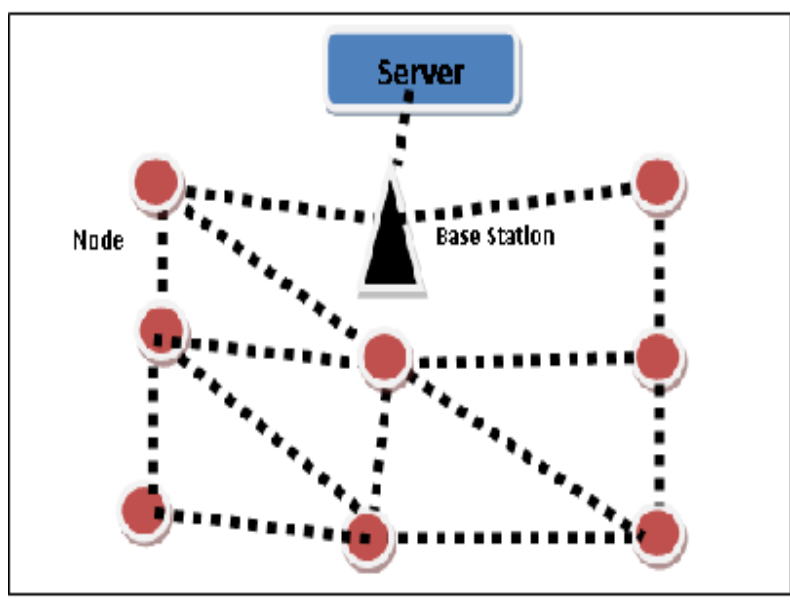

Figure 1: Wireless Sensor Network

Clustered Network Architecture: The basic objective on any routing protocol is to make the network useful and efficient. A cluster based routing protocol group's sensor nodes where each group of nodes has a $\mathrm{CH}$ or a gateway.

Sensed data is sent to the $\mathrm{CH}$ rather than send it to the $\mathrm{BS} ; \mathrm{CH}$ performs some aggregation function on data it receives then sends it to the BS where these data is needed. A clustered sensor network could also be classified as

- $\quad$ Single hop model

- Multi hop model

In a single hop network is one in which sensor nodes use single hopping to reach the cluster head. LEACH is an example of homogenous Single Hop model. Both LEACH and LEACH - C use one level clustering [13].

In a multi-hop network, nodes use multi-hopping to reach the cluster head. The extension of LEACH called M - LEACH or multi - hop LEACH is a good example of multi hop model [13]. 


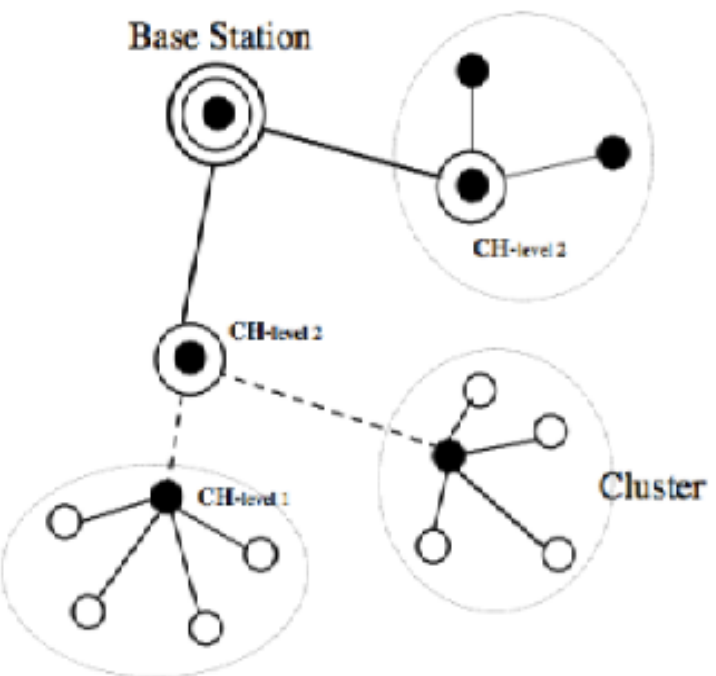
Figure 2: Multi-Hop Wireless Sensor Network
Architecture

In this paper, we propose a mechanism of selecting cluster heads based on the energy as well as the connectivity, probability parameters. The overall load of the network is balanced by balancing the load of cluster heads. A ratio based approach is used to divide the load among the clusters.

The remainder of the paper is organized as follows: Section 2 describes the architecture of wireless sensor network; section 3 specifies the related work in this field. Section 4 describes the work in detail along with its significance. Section 5 concludes the paper and discusses future work

\section{SYSTEM MODEL}

The energy limitation is one of the crucial factor that is required to consider for the construction of sensor network. In this network, each participating node in communication gives some energy loss. As the data is transmitted, received or forwarded some amount of energy is lost. Because of this, reducing the energy consumption is required in such network to improve the network life and performance. To perform the effective communication some network architecture is required. This kind of network is defined as the clustered network in which each cluster is defined with gateways specification. The energy effective communication is required to improve the network life. The proposed work is in same direction. Another important concept associated with sensor network is the aggregation. The aggregation is about to collect data from a node and combine the new information with it and forward to the next node. The process of aggregation is repeated on each node till the destination node not found. The aggregation is basically to convert the multicast communication to unicast and to reduce the network communication and to save the network energy that consumed during the communication process [8]. The proposed work defined an effective clustering architecture with effective election algorithm and the effective multi-hop communication within cluster and between the clusters. According to this presented model complete network is divided in small network area segments called clusters and each cluster is controlled by a gateways. The work is here focused on the selection of effective gateways under different constraints. The constraints are defined in terms of limit specification to the number of cluster members, number of clusters over the network and the energy specification. This improved algorithm will reduce the energy consumption and achieve the energy balancing over the network. Once the clusters are defined, the next work is to perform the communication over the cluster. For this communication three level communication is defined. The first level is defined with the lower limit within cluster. The nodes present in this range can perform direct communication with gateways. In second level, the multi-hop communication is performed by the cluster distance node. The third level communication is performed between the gateways to perform effective multi-hop communication to deliver the information to base station.

In this paper, a new cluster head specification approach is defined under the following specification

- The minimum and maximum number of clusters over the sensor network so that the uniform distribution will be performed.

- The minimum number of nodes that will be connected to the main cluster head at any instance of time. This is basically to perform the localization in sensor network. So that the chances of node loss will be reduced.

- The cluster head is defined with sensing range.

- The primary cluster head will be selected under the energy and the connectivity constraints.

- It must satisfy the probabilistic chances of setting a node as cluster head.

The energy is the main constraint for any sensor network. Each node in the network is defined with specific energy parameter (E). As the communication is performed, each participating node consumes some amount of energy.

\section{RELATED WORK}

Our work is motived by various research projects in sensor network domain. A lot of research work has been done in clustering field. Various approaches have been used to extend the lifetime of a sensor network. Many authors have proposed different methods to prolong the battery life of a sensor node. A Two Cluster Head Energy efficient Wireless Sensor Network (TCHE-WSN) Algorithm was put forward to save the node energy [7].An Energy Efficient Filtering Method has been proposed. In this approach, each node controls its verification probabilities for event reports, which depend on sending nodes. The number of recent valid reports and the false traffic ratio of each neighbor node are used to determine the verification probability for each node [6]. Computing Methodologies for Localization Techniques in Wireless Sensor Networks has been proposed. In this paper, the localization is one of the main protocols in WSN to find the position of unknown sensor nodes [14]. The published work does not take in to account the load balancing among clusters due to variable density of nodes in the system. If the load in the system is not balanced there can be clusters with high and low density. In such scenario the high density cluster head will be overloaded with the processing and communication and will consume its energy soon. The other cluster heads will sit idle and waste the resources of the system. Our approach proposes a load balanced network, where in the load is equally distributed among the cluster heads. The node degree is also considered while assigning the sensor node to the cluster [9]. 


\section{PROPOSED ALGORITHM FOR NODE DEGREE RATIO ANALYSIS}

In Multi-hop network architecture, the network is divided in to clusters, where each cluster has a cluster head and various sensor nodes. The sensor nodes sense the information from their surroundings and send this information to the Base station via cluster head. Cluster head combines the information collected from all its sensor nodes and send this to the base station in compressed form. The criteria of gateways/cluster head selection is one of the major tasks in this work. It is on the basis of three factors i.e. Distance, energy and connectivity constraints. Once the gateway is defined, the network is controlled by these gateways nodes. The distance restriction is defined for the internal and external cluser nodes. Finally, the route is generated to perform effective route based communication within clusters and between the clusters. The assumptions considered in this work are given as under

- All the nodes are distributed at random position.

- Each node is specified with energy parameters.

- The coverage range of cluster head as well as the base stations is also assumed

Algorithm (Nodes, $\mathrm{N}$ )

/* Nodes is the List N Nodes*/

\{

1. Perform the Randomized Localization of N Nodes over the Network.

2. For $\mathrm{i}=1$ to $\mathrm{N}$

[Initially All nodes are defined with energy

constraint and there is no gateway node in the network] \{

3. Set Nodes(i).Energy=Random

4. Set Nodes(i).ResidualEnergy=Random

5. Set Nodes(i).Type $=$ Normal

6. Set Nodes(i).ClusterHead=Null \}

7. Set energyThreshold, clusterThreshold and clusterNodeThreshold to generate a balanced clustered network

8. While $(\mathrm{R}<=$ MAX_ROUNDS $)$

[Perform the Communication for Defined Number of Rounds]

\{

9. For $\mathrm{i}=1$ to NumberofNodes [Process all Nodes to be set as Gateway] \{

10. If (Nodes(i).Energy $>$ Threshold And Probability(Node(i)) $>0$ And Node(i).Type=Normal) [Check the Initial Eligibility Criteria to set a Node as Gateway]

11. Find Number of Nodes in the SensingRange of Node(i) called M Nodes

12. If $(\mathrm{M}<$ clusterNodeThreshold $)$ \{

13. Print "UnderLoad Condition Meet cannot set as cluster head"

14. If $(\operatorname{Exist}(\operatorname{Nodes}(1: \mathrm{M}))="$ Gateway")

\{

15. Print "Other Gateway Exist in same Range"

16. For $\mathrm{j}=1$ to NoofNodes
17. $\operatorname{If}(\operatorname{Nodes}(\mathrm{j}) \cdot$ Type="Gateway" and Distance(Nodes(i),Nodes(j)) $<=$ SensingRange)

\{

18. Nodes(i).Degree=Nodes(i).Degree+1; \}

19. $\}$

20. Separate the Degree Nodes for Node i i.e. Degree 1,Degree 2 and Degree $M$

21. Ratio1=Count("Degree1")/ Count("Degree2")

22. Ratio2=Count("Degree2")/ Count("Degree3")

23. [Take the ratio for Degree 1, Degree II and Degree $M$ Nodes]

24. If (Ratio1 $>=2 \& \&$ Ratio $2>=2$ )

25. \{

26. Set Nodes(i).Type="Gateway"

27. $\}$

28. $\}$

\section{CONCLUSION AND FUTURE WORK}

In this present work effective gateway based architecture is defined to perform the load equalization and distribution in terms of network nodes. An ALGORITHM is proposed as a ratio analysis approach to perform equalizes distribution of gateway nodes. The presented work will generate the load balanced gateway network and provide the effective energy distribution over the network. This will improve the network life and the network communication.

In the existing work, the gateway selection is based on energy whereas in our paper, the connectivity ratio is also considered as the initial parameters so that the overload and under load gateway situation will be avoided. In the existing work, no ratio based node selection is defined in a gateway whereas in this present work ratio based analysis is defined while performing the gateway selection. Future work includes the implementation of this approach in MATLAB environment and comparison of this with the existing load balancing algorithms for performance analysis.

\section{REFERENCES}

[1] Gaurav Gupta and Mohamed Younis,"Performance Evaluation of Load balanced clustering of Wireless Sensor Networks"Telecommunications, 2003. ICT 2003. 10th International Conference on (Volume: 2 )

[2] A. Tarachand, Vikas Kumar, Abhishek Raj, Ashish Kumar and Prasanta k. Jana, "An Energy Efficient Load Balancing Algorithm for Cluster Based Wireless sensor networks."

[3] Ashutosh Kumar Singh," Minimization of Energy Consumption of wireless sensor networks using Fuzzy Logic2011 International Conference on Computational intelligence and communication systems.

[4] Darminder Singh Ghataoura," GAFO: Genetic Adaptive Fuzzy Hop Selection Scheme for Wireless Sensor Networks", IWCMC'09, June 21-24, 2009, Leipzig, GermanyACM 978-1-60558-569-7/09/06 (pp 376-380)

[5] ArpitaChakraborty," A Fuzzy based Trustworthy Route Selection Method usingLSRP in Wireless Sensor Networks (FTRSP)", CCSEIT-12, October 26-28, 2012, Coimbatore [Tamil nadu, India] ACM 978-1-4503-13100/12/10 (pp 413-419) 
[6] So Young Moon," Energy Efficient Filtering Method in Sensor Networks", ICUIMC-09, January 15-16, 2009, Suwon, S. Korea (pp 580-585)

[7] B.Meenakshi," Lifetime Extension of Wireless Sensor Network by selecting two Cluster Heads and hierarchical routing", ICACCI'12, August 3-5, 2012, Chennai, T Nadu, India. ACM 978-1-4503-1196-0/12/08 (pp 12541260)

[8] K. Akkaya and M. Younis, "A survey on routing protocols for wireless sensor networks," Ad Hoc Networks, vol. 3, no. 3, pp. 325-349, 2005.

[9] Vatsla chauhan and Sandeep Kumar,"A Node degree based ratio analysis approach to generate load balanced sensor network",2014, IJRIT Journal. Vol 2 Issue 5.

[10] Q. Li, M. D. Rosa, and D. Rus, "Distributed algorithms for guiding navigation across a Sensor network," in ACM MobiCom, 2003.

[11] H. Qi, S. Iyengar and K. Chakrabarty, "Multi-resolution data integration using mobile agents in distributed sensor networks," IEEE Trans. Syst., Man, Cybern, C, vol. 31, no. 3, pp. 383-391, Aug. 2001.

[12] S. Franklin and A. Graesser, "Is it an agent, or just a program?: A taxonomy for autonomous agents," in
Proceedings Third International Workshop on Agent Theories, Architectures, and Languages, J. G. Carbonell and J. Siekmann, Eds. New York: Springer-Verlag, 1996, vol. $1193 . \quad$ [Online]. Available: http://www.msci.memphis.edu/ franklin/AgentProg.html.

[13] H. Qi, Y. Xu, and X.Wang, "Mobile-agent-based collaborative signal and information processing in sensor networks," Proceedings of the IEEE, vol. 91, no. 8, pp. 1172-1183, 2003

[14] M Bala Krishna," Computing Methodologies for Localization Techniques in Wireless Sensor Networks", ICWET'11, February 25-26, 2011, Mumbai, Maharashtra, India. ACM 978-1-4503-0449-8/11/02 (pp 1024-1028)

[15] Bai, J., Zang, c., Wang, T., Yu, H.,"A Mobile AgentsBased Real-time Mechanism for Wireless Sensor Network Access on the Internet, "Proceedings of the 2006 IEEE International Conference on Information Acquisition, Weihai, Shandong, China, August 2006.

[16] J.Fernandez-Prieto," Poster Abstract: Architecture for redundant knowledge reduction in embedded Fuzzy Rule-Based Systems into Wireless Sensor Network nodes", IPSN'10, April 12-16, 2010, Stockholm, Sweden. ACM 978-1-60558-988-6/10/04. (pp 392-393). 